\title{
Risk of Hepatocellular Carcinoma in Patients with Hepatitis C Virus Who Achieved Sustained Virological Response
}

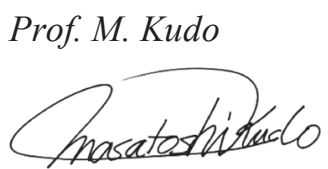

Editor Liver Cancer

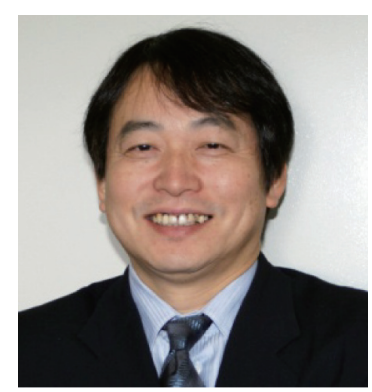

\section{Introduction}

The recent development of interferon-free direct-acting antivirals (DAAs) has had a significant impact on the already great advances made in the treatment of hepatitis C. Combination therapy with pegylated interferon, ribavirin and simeprevir has achieved a sustained virological response (SVR) rate of approximately $70 \%$ in patients with hepatitis C caused by the genotype Ib virus. Combination therapy using daclatasvir and asunaprevir, which was approved in Japan in September 2014, has increased the SVR rate to approximately 95\%. A clinical trial testing sofosbuvir-ledipasvir combination therapy (approved in September 2015 in Japan and October and November 2014 in the USA and EU, respectively) showed an SVR rate of nearly $100 \%$ in patients with genotype $1 \mathrm{~b}$ hepatitis $\mathrm{C}$ virus. Combination therapy with ombitasvir and paritaprevir, which has a powerful therapeutic effect (SVR rate $\geq 95 \%$ ), also became available in Japan from November 2015. Because interferon-based therapy is not possible in patients aged $>70$ years, in patients with cirrhosis, or in patients with a low platelet count, the above DAAs (which have extremely low rates of adverse effects) are extensively indicated. DAAs can be administered to almost all hepatitis $\mathrm{C}$ patients, including the elderly and those with compensated liver cirrhosis, and rapidly eradicate hepatitis $\mathrm{C}$ viruses; high SVR rates are achieved even in elderly cirrhotic patients with a high risk of liver cancer. Consequently, a new clinical problem has emerged, namely, the development of liver cancer after SVR [1-12]. Thus, two new unmet needs have arisen in the clinical setting: (1) finding the best possible way to assess the risk of liver cancer development after SVR is achieved and (2) establishing a liver cancer screening program for these patients $[13,14]$. 


\section{$\alpha$-Fetoprotein (AFP)}

It is well known that liver cancer can develop in elderly patients and patients with advanced fibrosis who have achieved SVR after interferon-based therapy [1-12]. Nationwide data reveal that $2.4 \%(109 / 4,542)$ of Japanese patients who achieved SVR developed liver cancer during an observation period of approximately 5.5 years [15]. The longest interval between SVR and cancer onset was more than 15 years. The same study also showed that advanced fibrosis, hypoalbuminemia before interferon treatment, and high baseline AFP levels were strongly associated with the development of hepatocellular carcinoma (HCC).

When treatment with DAAs became available, the initial concern was that the drugs would be inferior to interferon-based therapy in terms of reducing the risk of liver cancer development. However, now that several tens of thousands of patients with hepatitis $C$ have been treated with DAAs in Japan, it is clear that AFP levels, a surrogate marker of HCC development, drop to $\leq 10 \mathrm{ng} / \mathrm{ml}$ after achieving SVR, thereby dispelling this initial concern. These low AFP levels also suggest that both DAAs and interferon-based therapies will reduce the cancer risk.

A large-scale cohort study revealed that old age, advanced fibrosis, male gender, and high post-treatment AFP levels are risk factors for post-SVR HCC development [16]. In addition to high AFP levels, high alanine transaminase levels and platelet counts are reportedly risk factors for cancer development after treatment with interferon-based therapy, regardless of whether SVR is achieved [17-19].

Two different cut-offs have been suggested for AFP levels after interferon-based treatment: $6 \mathrm{ng} / \mathrm{ml}$ [17] and $10 \mathrm{ng} / \mathrm{ml}$ [19]. The risk of HCC development appears to be high in patients whose AFP levels remain $\geq 10 \mathrm{ng} / \mathrm{ml}$ after antiviral treatment (figs. 1 and 2). Regardless of whether SVR is achieved, surveillance needs to be continued in such patients to detect HCC at an early stage. It is recommended that elderly patients or patients with advanced fibrosis who have post-treatment AFP levels $\geq 10 \mathrm{ng} / \mathrm{ml}$ be identified as a high-intermediate risk group. This group should be tested for three types of tumor marker (AFP, prothrombin induced in the absence of vitamin K [PIVKA-II], and the lectin-binding fraction of AFP [AFPL3]) and undergo ultrasonography every 6-12 months.

\section{Mac-2 Binding Protein Glycosylation Isomer (M2BPGi)}

The carbohydrate M2BPGi is a serum marker of liver fibrosis [20] that was developed by Sysmex Corp. (Kobe, Japan) and a team led by Dr. Hisashi Narimatsu (Research Centre for Medical Glycoscience, National Institute of Advanced Industrial Science and Technology, Tokyo, Japan). Carbohydrate epitopes developed as markers of liver fibrosis have been used to diagnose fibrosis in patients with hepatitis $\mathrm{C}$, hepatitis B, and non-alcoholic fatty liver disease. In recent years, their utility as markers of HCC development in hepatitis C patients has been of particular interest. The rate of HCC in patients with hepatitis C gradually increases from $0.5 \%$, to $1.5 \%, 3 \%, 5 \%$, and $8 \%$ as hepatic fibrosis progresses from stage $\mathrm{F} 0$ to $\mathrm{F} 1$, F2, F3, and F4, respectively. Yamasaki et al. measured M2BPGi levels in 707 patients with chronic hepatitis $\mathrm{C}$ who had undergone liver biopsy and found that progression of fibrosis correlated well with M2BPGi levels [21]. More precisely, the proportion of patients with F0F1 and F4 was clearly different in those with an M2BPGi cutoff index (COI) $\leq 1(80.6 \%$ and $1.3 \%$, respectively) and in those with an $\mathrm{M} 2 \mathrm{BPGi} \mathrm{COI} \geq 8$ (3.1\% and $78.1 \%$, respectively). Furthermore, the cumulative incidence of HCC increased as M2BPGi levels increased regardless of fibrosis stage (fig. 3) [21]. 


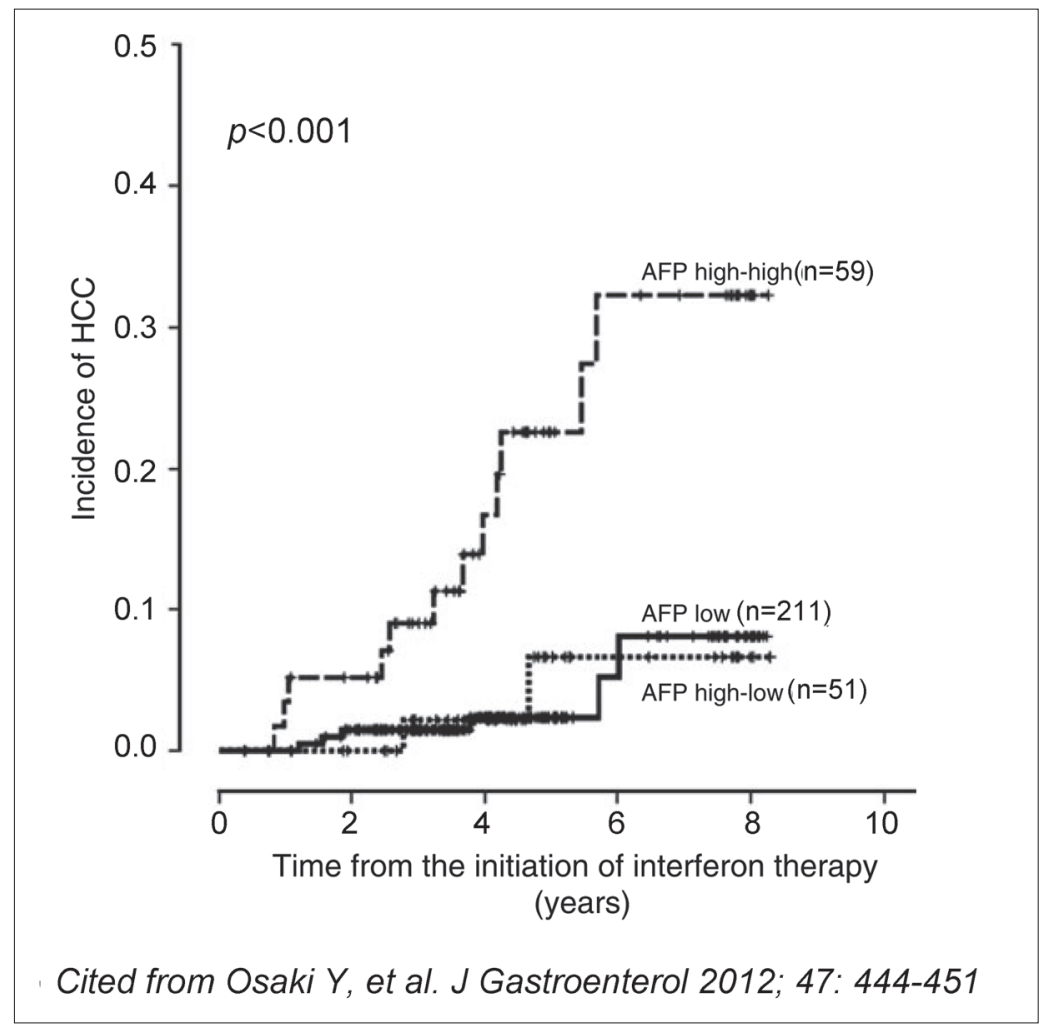

Fig. 1. Kaplan-Meier estimates of the incidence of HCC after interferon therapy for hepatitis C. The solid line denotes the AFP-low group (AFP levels before interferon therapy $<10 \mathrm{ng} / \mathrm{ml}$ ), the dotted line denotes the AFP high-low group (baseline AFP levels $\geq 10 \mathrm{ng} / \mathrm{ml}$, average AFP integration level $<10 \mathrm{ng} / \mathrm{ml}$ ), and the dashed line denotes the AFP high-high group (both baseline and average AFP integration levels $\geq 10$ $\mathrm{ng} / \mathrm{ml}$ ). Reproduced with permission from Osaki Y, et al. [19]

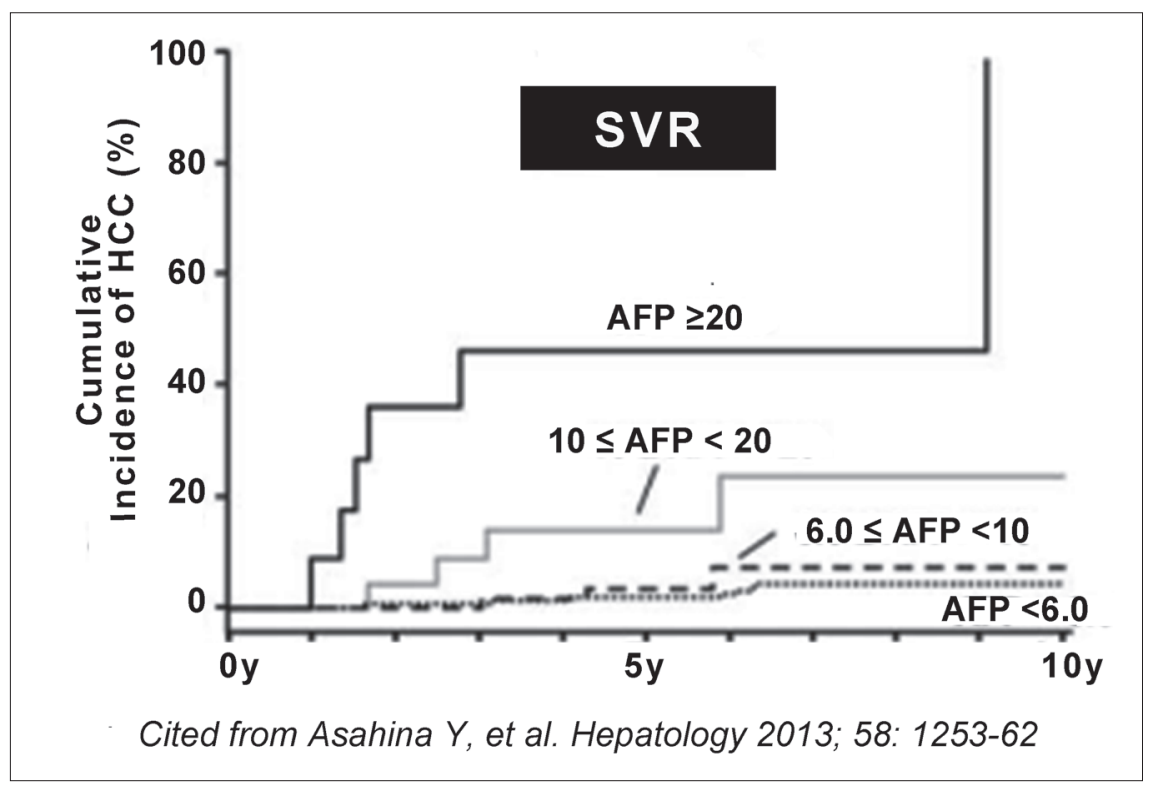

Fig. 2. Cumulative incidence of HCC in chronic hepatitis $C$ patients according to post-interferon treatment AFP level. Patients with SVR after treatment of hepatitis $C$ were stratified according to post-IFN treatment AFP levels (log-rank test: $\mathrm{p}<0.0001$ ). Reproduced with permission from Asahina Y, et al. [17] 


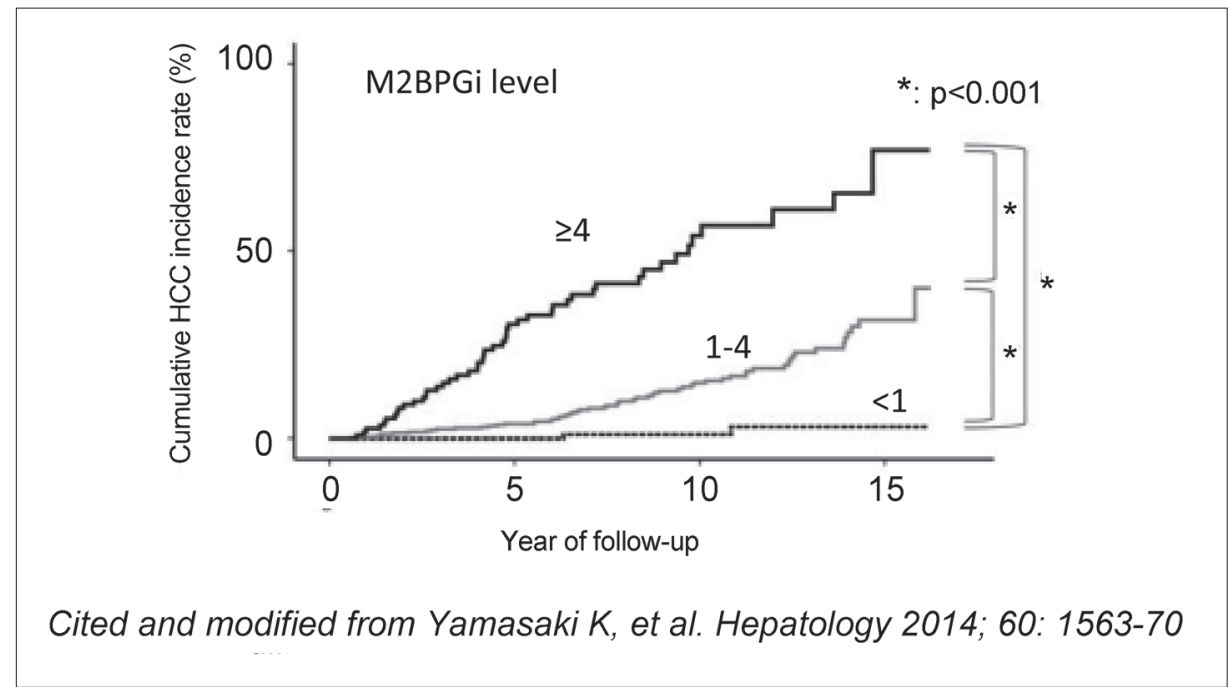

Fig. 3. Cumulative incidence of HCC according to M2BPGi levels in patients with hepatitis C, as analyzed using the Kaplan-Meier method. Black solid, gray solid, and dotted lines denote stratified M2BPGi levels $\geq 4,1-4$, and $<1$, respectively. The HCC incidence differed significantly between the three groups $(\mathrm{p}<0.001$, log-rank test) and increased with increasing M2BPGi levels. Reproduced with permission from Yamasaki K, et al. [21]

Multivariate analysis using the Cox proportional hazards model was performed to identify factors contributing to the development of HCC. Fibrosis stage (F0-F1, F2, F3, F4), AFP $(<6,6-20, \geq 20 \mathrm{ng} / \mathrm{ml})$, age ( $<57$ or $\geq 57$ years old), interferon therapy (no therapy versus SVR) were significantly associated with the risk of HCC. It should be noted that fibrosis stage F4 versus stages F0-F1 was the only significant factor indicative of increased risk of HCC development as far as the fibrosis stage is concerned, whereas an M2BPGi COI of 1-4 (hazard ratio $5.2, p=0.029$ ) and of $\geq 4$ (hazard ratio $8.3, p=0.007$ ) were significant factors versus an M2BPGi COI $\leq 1$. This finding indicates that M2BPGi is a more useful factor for predicting the development of HCC than the fibrosis stage is [21].

Analysis of the time-dependent area under the receiver operating characteristic (AUROC) curve was also used to compare the predictive performances of M2BPGi, AFP, and platelet count. M2BPGi levels (based on AUROC analysis that examined the period from year 1 to year 13 after liver biopsy) were superior to AFP levels and platelet counts for predicting HCC development in years 3 and 5.

The above data were derived by comparing the incidence of HCC development in the overall cohort of patients with chronic hepatitis C. A similar approach could be used to predict the risk of cancer development in the subgroup of hepatitis C patients who achieved SVR after antiviral treatment.

Sasaki et al. measured M2BPGi levels in 238 patients with hepatitis $\mathrm{C}$ and found that they were significantly higher in the 16 patients (6.8\%) who developed HCC after achieving SVR than in the remaining 222 patients (93.2\%) who did not. Multivariate analysis also revealed that the M2BPGi level is an independent predictive factor for HCC development (fig. 4) [22].

Tamaki et al. used a cumulative scoring system in which an annual change in the M2BPGi level of $\geq 0.3$, an AFP level of $\geq 10 \mathrm{ng} / \mathrm{ml}$, and an M2BPGi COI of $\geq 4.2$ were individually counted as one point (or as zero points when they were less than these values). They found that HCC development was rarely confirmed in patients with a cumulative score of zero, whereas the risk of HCC development gradually increased as the cumulative score increased from 1 


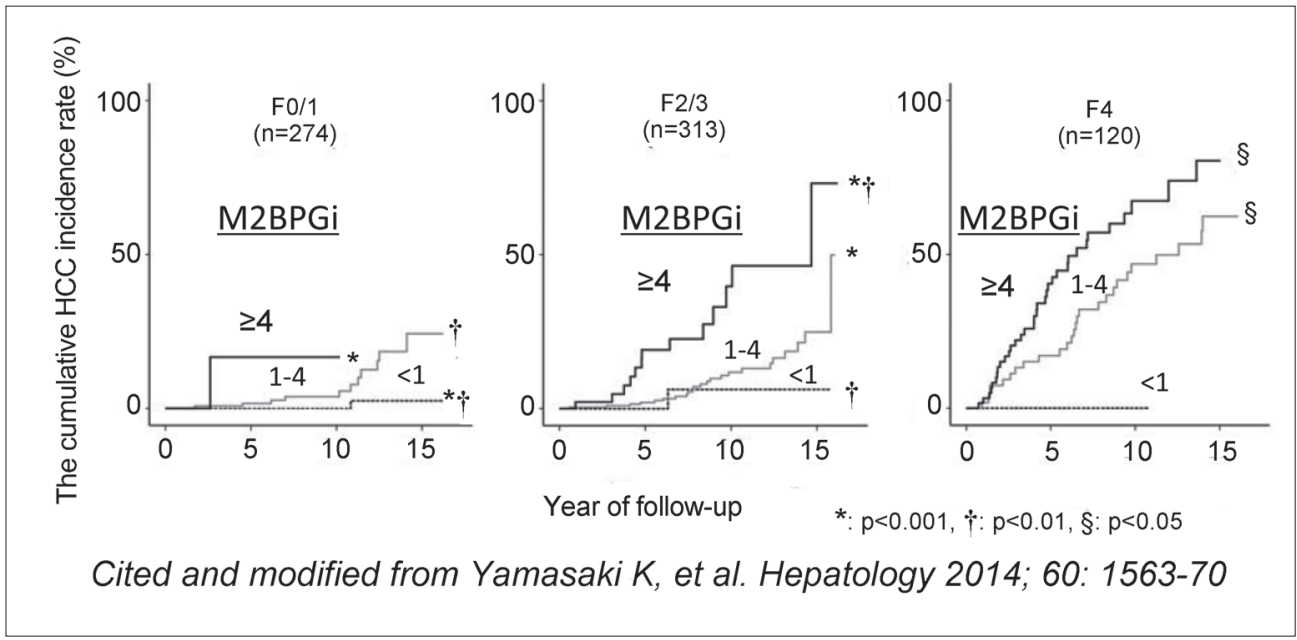

Fig. 4. Cumulative incidence of HCC in patients with hepatitis $C$ according to M2BPGi levels stratified according to the fibrosis stage as analyzed using the Kaplan-Meier method. Black solid, gray solid, and dotted lines denote stratified M2BPGi levels $\geq 4,1-4$, and $<1$, respectively. The HCC incidence increased with increasing M2BPGi levels. Reproduced with permission from Yamasaki K, et al. [21]

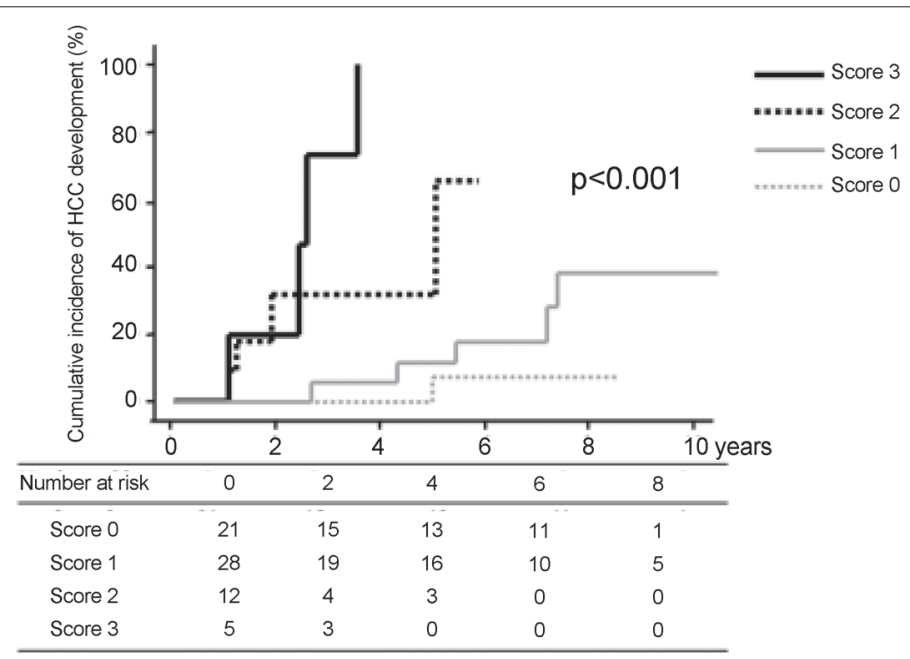

Scoring: $\mathrm{M} 2 \mathrm{BPGi} \geq 4.2, \triangle \mathrm{M} 2 \mathrm{BPGi} / \mathrm{year} \geq 0.3$, and $\mathrm{AFP} \geq 10 \mathrm{mg}$ were individually counted as one point

Cited and modified from Tamaki N, et al. Hepatol Res. 2015; 45 :E82-8

Fig. 5. Association between the risk score and the cumulative incidence of HCC development in chronic hepatitis $\mathrm{C}$ patients. M2BPGi $\geq 4.2, \Delta \mathrm{M} 2 \mathrm{BPGi} / \mathrm{year} \geq 0.3$, and AFP $\geq 10 \mathrm{ng} / \mathrm{ml}$ each contributed one point to the overall score. M2BPGi $<4.2, \Delta \mathrm{M} 2 \mathrm{BPGi} /$ year $<0.3$, and AFP $<10 \mathrm{ng} / \mathrm{ml}$ each contributed zero points to the overall score. Patients were classified into four groups according to their total score $(0,1,2$, or 3$)$. Reproduced with permission from Tamaki N, et al. [23]

to 3 (fig. 5) [23]. Clearly, blood tests measuring the above three factors provide a certain level of prediction regarding post-SVR cancer risk. This finding also supports the notion that the M2BPGi level is useful for predicting HCC development in those with chronic hepatitis $\mathrm{C}$ who have achieved SVR after antiviral therapy. 


\section{Conclusion}

In the future, M2BPGi will play an extremely important role in predicting HCC development in hepatitis C patients who achieve SVR after antiviral therapy. This factor can be used alongside post-treatment AFP levels, for which a consensus on its predictive utility has almost been reached. Additionally, a scoring system that combines AFP and M2BPGi levels appears to be beneficial. The growing clinical problems associated with the rapidly emerging era of DAA therapy may be reduced by considering post-treatment AFP and M2BPGi levels in addition to old age, male gender, and low platelet count when analyzing the post-SVR HCC risk in patients with chronic hepatitis C. This approach will also be useful for introducing different screening intervals determined according to the results of individual risk analysis.

Although evidence indicating that both AFP and M2BPGi levels in hepatitis C patients after achievement of SVR are important for assessing the post-SVR risk of developing HCC has come only from Japan, these two serum markers will be of particular significance when developing screening strategies for post-SVR HCC development. It is anticipated that M2BPGi testing will soon be available in the clinical setting in other parts of the world, including Asian and Western countries.

\section{References}

1 Asahina Y, Tsuchiya K, Tamaki N, Hirayama I, Tanaka T, Sato M, Yasui Y, Hosokawa T, Ueda K, Kuzuya T, Nakanishi H, Itakura J, Takahashi Y, Kurosaki M, Enomoto N, Izumi N: Effect of aging on risk for hepatocellular carcinoma in chronic hepatitis C virus infection. Hepatology 2010;52:518-527.

2 Nagaoki Y, Aikata H, Miyaki D, Murakami E, Hashimoto Y, Katamura Y, Azakami T, Kawaoka T, Takaki S, Hiramatsu A, Waki K, Imamura M, Kawakami Y, Takahashi S, Chayama K: Clinical features and prognosis in patients with hepatocellular carcinoma that developed after hepatitis $\mathrm{C}$ virus eradication with interferon therapy. J Gastroenterol 2011;46:799-808.

3 Ohki T, Tateishi R, Sato T, Masuzaki R, Imamura J, Goto T, Yamashiki N, Yoshida H, Kanai F, Kato N, Shiina $\mathrm{S}$, Yoshida H, Kawabe T, Omata M: Obesity is an independent risk factor for hepatocellular carcinoma development in chronic hepatitis C patients. Clin Gastroenterol Hepatol 2008;6:459-464.

4 Kobayashi S, Takeda T, Enomoto M, Tamori A, Kawada N, Habu D, Sakaguchi H, Kuroda T, Kioka K, Kim SR, Kanno T, Ueda T, Hirano M, Fujimoto S, Jomura H, Nishiguchi S, Seki S: Development of hepatocellular carcinoma in patients with chronic hepatitis $\mathrm{C}$ who had a sustained virological response to interferon therapy: A multicenter, retrospective cohort study of 1124 patients. Liver Int 2007;27:186-191.

5 Hirakawa M, Ikeda K, Arase Y, Kawamura Y, Yatsuji H, Hosaka T, Sezaki H, Akuta N, Kobayashi M, Saitoh S, Suzuki F, Suzuki Y, Kumada H: Hepatocarcinogenesis following HCV RNA eradication by interferon in chronic hepatitis patients. Intern Med 2008;47:1637-1643.

6 Tanaka A, Uegaki S, Kurihara H, Aida K, Mikami M, Nagashima I, Shiga J, Takikawa H: Hepatic steatosis as a possible risk factor for the development of hepatocellular carcinoma after eradication of hepatitis $\mathrm{C}$ virus with antiviral therapy in patients with chronic hepatitis C. World J Gastroenterol 2007;13:5180-5187.

7 Tokita H, Fukui H, Tanaka A, Kamitsukasa H, Yagura M, Harada H, Okamoto H: Risk factors for the development of hepatocellular carcinoma among patients with chronic hepatitis $C$ who achieved a sustained virological response to interferon therapy. J Gastroenterol Hepatol 2005;20:752-758.

8 Ikeda M, Fujiyama S, Tanaka M, Sata M, Ide T, Yatsuhashi H, Watanabe H: Risk factors for development of hepatocellular carcinoma in patients with chronic hepatitis $\mathrm{C}$ after sustained response to interferon. J Gastroenterol 2005;40:148-156.

9 Iwasaki Y, Takaguchi K, Ikeda H, Makino Y, Araki Y, Ando M, Kobashi H, Kobatake T, Tanaka R, Tomita M, Senoh T, Kawaguchi M, Shimoe T, Manabe K, Kita K, Shimamura J, Sakaguchi K, Shiratori Y: Risk factors for hepatocellular carcinoma in hepatitis $\mathrm{C}$ patients with sustained virologic response to interferon therapy. Liver Int 2004;24:603-610.

10 Makiyama A, Itoh Y, Kasahara A, Imai Y, Kawata S, Yoshioka K, Tsubouchi H, Kiyosawa K, Kakumu S, Okita K, Hayashi N, Okanoue T: Characteristics of patients with chronic hepatitis C who develop hepatocellular carcinoma after a sustained response to interferon therapy. Cancer 2004;101:1616-1622.

11 Kasahara A, Hayashi N, Mochizuki K, Takayanagi M, Yoshioka K, Kakumu S, Iijima A, Urushihara A, Kiyosawa K, Okuda M, Hino K, Okita K, Osaka Liver Disease Study Group: Risk factors for hepatocellular carcinoma and its incidence after interferon treatment in patients with chronic hepatitis C. Hepatology 1998;27:1394-1402. 
12 Dohmen K, Kawano A, Takahashi K, Shigematsu H, Tanaka H, Haruno M, Yanagita K, Ichiki Y, Mori T, Hayashida K, Shimoda S, Ishibashi H, Nomura H: The incidence and risk factors for the development of hepatocellular carcinoma after peginterferon plus ribavirin therapy for chronic hepatitis C. Hepatogastroenterology 2013;60:2034-2038.

13 Kudo M: Surveillance, diagnosis, treatment, and outcome of liver cancer in Japan. Liver Cancer 2015;4:3950.

14 Kudo M: Clinical practice guidelines for hepatocellular carcinoma differ between Japan, United States, and Europe. Liver Cancer 2015;4:85-95.

15 Sato A, Sata M, Ikeda K, Kumada T, Izumi N, Asahina Y, Osaki Y, Chayama K, Kaneko S, Sakai A, Onji M, Hiasa Y, Omura T, Ozeki I, Yokosuka O, Shiina S, Itsubo M, Nishiguchi S, Hirano K, Ide T, Sakisaka S, Yamasaki T, Hidaka I, Tanaka M, Kim SR, Ichida T: Clinical characteristics of patients who developed hepatocellular carcinoma after hepatitis $\mathrm{C}$ virus eradication with interferon therapy: current status in Japan. Intern Med 2013;52:2701-2706.

16 Nagaoki Y, Aikata H, Nakano N, Shinohara F, Nakamura Y, Hatooka M, Morio K, Kan H, Fujino H, Kobayashi T, Fukuhara T, Masaki K, Ono A, Nakahara T, Kawaoka T, Miki D, Tsuge M, Hiramatsu A, Imamura M, Takahashi S, Kawakami Y, Ochi H, Chayama K, Hiroshima Liver Study Group: Development of hepatocellular carcinoma in patients with hepatitis $\mathrm{C}$ virus infection who achieved sustained virological response following interferon therapy: a large-scale, long-term cohort study. J Gastroenterol Hepatol 2015 Nov 19. doi: 10.1111/jgh.13236. [Epub ahead of print] .

17 Asahina Y, Tsuchiya K, Nishimura T, Muraoka M, Suzuki Y, Tamaki N, Yasui Y, Hosokawa T, Ueda K, Nakanishi H, Itakura J, Takahashi Y, Kurosaki M, Enomoto N, Nakagawa M, Kakinuma S, Watanabe M, Izumi $\mathrm{N}$ : $\alpha$-Fetoprotein levels after interferon therapy and risk of hepatocarcinogenesis in chronic hepatitis $\mathrm{C}$. Hepatology 2013;58:1253-1262.

18 Oze T, Hiramatsu N, Yakushijin T, Miyazaki M, Yamada A, Oshita M, Hagiwara H, Mita E, Ito T, Fukui H, Inui Y, Hijioka T, Inada M, Katayama K, Tamura S, Yoshihara H, Inoue A, Imai Y, Hayashi E, Kato M, Miyagi T, Yoshida Y, Tatsumi T, Kasahara A, Hamasaki T, Hayashi N, Takehara T, Osaka Liver Forum: Post-treatment levels of $\alpha$-fetoprotein predict incidence of hepatocellular carcinoma after interferon therapy. Clin Gastroenterol Hepatol 2014;12:1186-1195.

19 Osaki Y, Ueda Y, Marusawa H, Nakajima J, Kimura T, Kita R, Nishikawa H, Saito S, Henmi S, Sakamoto A, Eso Y, Chiba T: Decrease in alpha-fetoprotein levels predicts reduced incidence of hepatocellular carcinoma in patients with hepatitis C virus infection receiving interferon therapy: a single center study. J Gastroenterol 2012;47:444-451.

20 Kuno A, Ikehara Y, Tanaka Y, Ito K, Matsuda A, Sekiya S, Hige S, Sakamoto M, Kage M, Mizokami M, Narimatsu H: A serum "sweet-doughnut" protein facilitates fibrosis evaluation and therapy assessment in patients with viral hepatitis. Sci Rep 2013;3:1065. doi: 10.1038/srep01065. Epub 2013 Jan 15

21 Yamasaki K, Tateyama M, Abiru S, Komori A, Nagaoka S, Saeki A, Hashimoto S, Sasaki R, Bekki S, Kugiyama Y, Miyazoe Y, Kuno A, Korenaga M, Togayachi A, Ocho M, Mizokami M, Narimatsu H, Yatsuhashi H: Elevated serum levels of Wisteria floribunda agglutinin-positive human Mac-2 binding protein predict the development of hepatocellular carcinoma in hepatitis C patients. Hepatology 2014;60:1563-1570.

22 Sasaki R, Yamasaki K, Abiru S, Komori A, Nagaoka S, Saeki A, Hashimoto S, Bekki S, Kugiyama Y, Kuno A, Korenaga M, Togayachi A, Ocho M, Mizokami M, Narimatsu H, Ichikawa T, Nakao K, Yatsuhashi H: Serum wisteria floribunda agglutinin-positive mac-2 binding protein values predict the development of hepatocellular carcinoma among patients with chronic hepatitis $\mathrm{C}$ after sustained virological response. PLoS ONE 2015;10:e0129053.

23 Tamaki N, Kurosaki M, Kuno A, Korenaga M, Togayachi A, Gotoh M, Nakakuki N, Takada H, Matsuda S, Hattori N, Yasui Y, Suzuki S, Hosokawa T, Tsuchiya K, Nakanishi H, Itakura J, Takahashi Y, Mizokami M, Narimatsu H, Izumi N: Wisteria floribunda agglutinin positive human Mac-2-binding protein as a predictor of hepatocellular carcinoma development in chronic hepatitis C patients. Hepatol Res 2015;45:E82-88. 